\title{
EXPLOITING DATA DIVERSITY AND MULTIUSER DIVERSITY IN NONCOOPERATIVE MOBILE INFOSTATION NETWORKS
}

\author{
Wing Ho Yuen \\ Roy D. Yates \\ Siun-Chuon Mau \\ WINLAB, Rutgers University \\ andyyuen@winlab.rutgers.edu ryates@winlab.rutgers.edu siunmau@winlab.rutgers.edu
}

\begin{abstract}
In wireless networks, it is often assumed that all nodes cooperate to relay packets for each other. Although this is a plausible model for military or mission based networks, it is unrealistic for commercial networks and future pervasive computing environments. We address the issue of noncooperation between nodes in the context of content distribution in mobile infostation networks. We assume all nodes have common interest in all files cached in the fixed infostations. In addition to downloading files from the fixed infostations, nodes act as mobile infostations and exchange files when they are in proximity. We stipulate a social contract such that an exchange occurs only when each node can obtain something it wants from the exchange. Our social contract enables much higher system efficiency compared to downloading from fixed infostations only while not requiring true cooperation among nodes. We show by analysis and simulations that network performance depends on the node density, mobility and the number of files that are being disseminated. Our results point to the existence of data diversity for mobile infostation networks. The achievable throughput increases as the number of files of interest to all users increases. We have also extended the common interest model to the case where nodes have dissimilar interests. Our simulation results show that as mobile nodes change from having identical interests to mutually exclusive interests, the network performance degrades dramatically. We propose an alternative user strategy when nodes have partially overlapping interests and show that the network capacity can be significantly improved by exploiting multiuser diversity inherent in mobile infostation networks. We conclude that data diversity and multiuser diversity exist in noncooperative mobile infostation networks and can be exploited.
\end{abstract}

\section{INTRODUCTION}

In generic mobile ad hoc networks, nodes communicate with each other through multihop routing. However, the achievable capacity in these networks is low as demonstrated by simulation studies [1], [2]. Although rate adaptation [7] or power control [11] techniques have shown demonstrative improvement on network capacity, it is unlikely that these measures will increase capacity further by several orders of magnitude. Indeed, [6] showed that the asymptotic throughput capacity of a wireless multihop network goes to zero as the number of nodes tends to infinity, even under the assumption of optimal scheduling and power control.

Recently, new mobile networking architectures have been proposed that exploit node mobility to achieve large network capacity. Instead of using multihop routing, networking is brought about by node mobility. In [5], nodes are connected intermittently when they are in proximity. It was shown in [5] that with a two hop relay model, the steady-state pernode throughput scales with the number of nodes. Whereas
[5] considered unicast communication, [10] considered content distribution using single hop multicast. In order to expedite data dissemination, a node also relays packets for other nodes if it has not done so for some time. The above works assume that nodes cooperate to relay packets for each other. Although typical in the wireless networking literature, this assumption is often unrealistic. When a node relays a packet for some other nodes, it expends its own bandwidth and power resources. A node therefore has no immediate incentive to forward packets for others.

In this paper we address the issue of noncooperation in the context of a mobile infostation network for movie downloading. All nodes are subscribers to a movie content distribution network. A movie is divided into $K$ files which are then cached in a network of fixed infostations, access points providing pockets of high-speed short-range coverage [3]. When a node comes close to an infostation, files can be downloaded. In an entirely noncooperative network, this would be the only mechanism for file dissemination. It only uses the high-speed channel between an infostation and a node near it, while wasting all the equally excellent channels between closely located nodes. A more efficient system would have any two nodes in proximity to act as mobile infostations to exchange copies of their files. When there are many nodes, a node obtains most of the files from node-to-node file exchanges. Data dissemination is thus distributed to all nodes and all locations in the network.

It is possible to allow file exchanges among mobile nodes while keeping the network essentially noncooperative by stipulating the following social contract for all nodes in the network. When two nodes meet, they inspect the file contents of each other. If each node identifies a file that it wants, a bilateral file exchange takes place. Conversely, if either of the nodes cannot find a file it wants, no file exchange takes place since that node has no immediate incentive to transmit a file to the other.

We have shown by analysis and simulations that the networking performance of this file exchange mechanism depends on node mobility and density. More importantly, we find that both fairness and throughput of the network improve as the number of files in the network increases. We identify this phenomenon as a new form of diversity. Traditional communication diversity techniques exploit the variations of signal strength over temporal, spatial and frequency domains. Data diversity, on the other hand, arises due to the enlargement 
of individuals' preferences of data, and is a consequence of the assumption of noncooperation among the nodes. We conjecture that data diversity has important ramifications in the performance of other networking contexts such as multihop ad hoc networks.

We have also extended the common interest model to the case where each node has dissimilar interest. This is applicable to the contexts in which multiple movies or TV shows are cached in the infostations. When nodes have mutually exclusive or partially overlapping interests, network performance degrades drastically. We have identified two user strategies for the dissimilar interest model. Our simulation results show that network capacity can be significantly improved by exploiting multiuser diversity inherent in mobile infostation networks.

The rest of the paper is organized as follows. In section II, we describe the system model. Section III is devoted to performance analysis, and the results are verified by simulations in section IV. We describe a new form of diversity - data diversity in section V. In section VI, we extend our common interest model to the case where nodes have partially overlapping interests. Simulation results of two user strategies are discussed. The results are interpreted further as a form of multiuser diversity in section VII. Finally, conclusions are drawn in section VIII.

\section{SySTEM MODEL}

This work is largely motivated by [5] which employed a signal to interference ratio (SIR) based link quality model to demonstrate the that $N$ nodes in a region could maintain $O(N)$ simultaneous transmissions with acceptable SIR. However, in this work, we look to employ a simpler communication model in order to demonstrate the effect of the social contract on content distribution. As shown in Figure 1, the geography consists of $L$ discrete locations in a square grid with an infostation at the center of the grid. The infostation cache holds the $K$ files of a movie. We assume the geography wraps around at each boundary, effectively creating a toroidal grid. We refer to this $L$ node wraparound grid with one infostation and $L-1$ regular locations as a block. A block is intended to mimic a typical multi-infostation network in which an infinite grid of infostations populate an infinite plane. The number of locations $L$ relative to the single infostation serves to characterize the density of fixed infostations over the terrain.

The $L$ location grid is populated with $N$ nodes with independent mobility processes. In our simulation experiments, we assume that time is discretized such that at each unit of time, each node randomly and independently moves in one of the four directions with equal probability $q=0.25$. When two or more nodes are at the same location at the same time, we say those nodes are neighbors.

In our communication model, each node either downloads files from an infostation or exchanges files with a neighbor. At the infostation, only file downloading is allowed. At any other locations, file exchanges between mobile nodes are permitted.

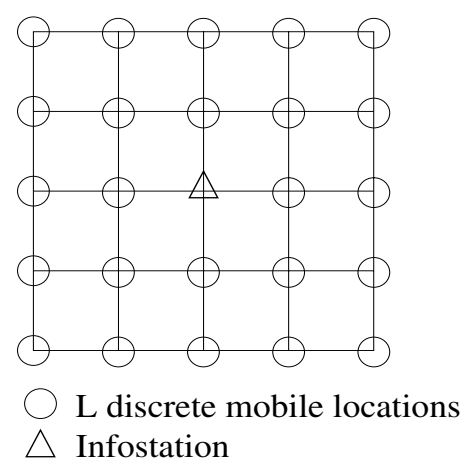

Fig. 1. Illustration of the network model.

Given a particular radio bandwidth, the size of a file is chosen such that the time a node occupies a location allows for either a bilateral file exchange between neighbors at a regular location or for two files to be downloaded from the infostation.

There are two factors that impact data dissemination. First there is a transmission concurrency constraint at each location. If there is more than one node at the infostation, contention is resolved by randomly picking one node for downloading. Similarly, when there are more than two neighbors at a location, two of the neighbors are randomly picked to perform a file exchange. Second, the probability of exchange is dictated by the user strategy which also consists of two parts. The user strategy must determine first whether to exchange files according to a social contract. Specifically, a node may want to exchange for a file because it is genuinely interested in that file. Alternatively, a node may want to exchange for a popular file, which is then used to facilitate future file exchanges. Thus even if a node cannot obtain a file of genuine interest, it may exchange for a file that it does not have. The user strategy then must specify which file should be picked from the other node. In the first part of this paper, however, there is no distinction between the above models. Since all nodes have common interest in downloading the files of a popular movie, each node is genuinely interested in every file it does not have. In section VI, we extend the common interest model to the case where nodes have dissimilar interests that are partially overlapping. In that case, the network performance is dependent on the choice of the above models.

After two neighbors agree to exchange files, each downloads one file from the other. In an encounter in which there are multiple files of interest, a node must decide which file to download. Two strategies are examined in this paper. For the random strategy, a node randomly selects a file it does not have from the neighbor node. Similarly, a node randomly selects two files that he does not have for downloading at an infostation. For comparison, we also consider a greedy strategy which assumes that each node has full knowledge of the circulation of each file within the network. For an infostation download or a neighbor exchange, a node picks the file that is the least circulated among all files it does not have. This strategy is greedy since it maximizes the probability of 
exchange $P_{E}$ between two arbitrary nodes in a static snapshot.

We note that the selection of two arbitrary nodes for file exchange is suboptimal. Under the social contract the two selected nodes may not perform file exchange. A practical node selection protocol should avoid this by scheduling transmissions only to the node pair with an exchange agreement. The random selection of nodes in this paper is used to facilitate performance analysis and provide a lower performance bound to an ideal node selection scheme. On the other hand, the social contract implicitly assumes there are no misbehaved nodes. Each node makes no false claim on the files it possesses and ensures the integrity of all its disseminated files. The social contract provides a framework for studying non-cooperation between nodes. In a practical file exchange protocol, additional security mechanisms must be added to ensure the integrity of the files being exchanged.

The proposed content distribution network admits a number of performance metrics to describe how quickly files are disseminated. We define $T_{1}$ as the time when $80 \%$ of the nodes get all of the files. A network operator is interested in this quantity, which is related to the networking efficiency and the revenue generated from the network. We define $T_{2}$ as the time when all nodes get $80 \%$ of the files. A network subscriber, on the other hand, will be interested in $T_{2}$, which is related to fairness and perhaps will influence his willingness to pay. We also define $T_{3}$ as the time for all nodes to get all the files. Finally $T_{4}$ is defined as the time for an arbitrary node to obtain all files. An analytical expression for $E\left[T_{4}\right]$ is obtained in the next section.

We also evaluate the network performance in terms of throughput $C_{i}$, which characterizes the average rate of file downloading per node. This is defined in terms of the networking time $T_{i}$ and is given by $C_{i} \triangleq K / E\left[T_{i}\right]$, for $i=1,2,3,4$. The units of $C_{i}$ are files per node per unit time. Note that we can view the distribution to a particular node of movies over time as a renewal process in which the renewal period equals $T_{4}$, the time required for the node to obtain one movie. Since the node obtains a reward of $K$ files in each renewal period, renewal-reward theory assures that the expected rate at which the node obtains files is precisely $C_{4}$ [12].

\section{Performance Analysis}

When two or more mobile nodes are at the same location, a two-step process determines whether a file exchange occurs. First, the nodes at that location follow a radio access protocol to determine which pair of nodes will attempt a file exchange. We use the term access to refer to the event that a node gets to be one of a pair of nodes that examines the files carried by the other. Under some simplifying assumptions, we will see that at a regular location the access probability is given by a constant $\beta$, that depends on the number of nodes $N$ and locations $L$ in the block. For a pair of nodes chosen in the access phase, the exchange probability $P_{E}$ denotes the probability that the two nodes can exchange files under the terms of the social contract.
The exchange probability will depend on the file contents in each node, which in turn depends on the user strategy.

In this section we provide a simple approximate analysis of $\beta$ and $P_{E}$. We then develop a simple Markov chain model to obtain the expected networking time $E\left[T_{4}\right]$ and the corresponding throughput $C_{4}$ for each node. We make the following key assumptions:

- Memoryless Uniform Mobility In each time unit, each node is randomly and independently at any of the $L$ locations with probability $p=1 / L$.

- Independent Uniform Content Distribution Given that node $i$ has obtained $l_{i}$ files, all combinations of $l_{i}$ out of $K$ files are equiprobable, independent of the files held by all other nodes.

It is not hard to see that these assumptions are inconsistent with the system model of section II. In particular, when the number of locations is small and mobility is limited, nodes are likely to be neighbors frequently and have highly correlated content. Nevertheless, our simulation results agrees closely with the analytical results, indicating that these assumptions work well in systems with moderately large number of files $K=500$ and reasonable mobility $q=0.25$.

Due to the transmission concurrency constraint, the maximum number of simultaneous transmissions in the block equals $L$, the number of locations. For a given number of locations, it should be apparent that there is an optimum number of nodes $N$ such that the access probability is maximized. If the number of nodes in the network is small, the spatial transmission concurrency is not fully utilized. Similarly, if there are too many nodes in the block, only a fraction of nodes could schedule transmissions in the $L$ possible locations.

Given a particular node at a given location, memoryless mobility implies that the number of other neighbors at that location is a random variable $J$ with the binomial distribution

$$
P[J=j]=\left(\begin{array}{c}
N-1 \\
j
\end{array}\right) p^{j}(1-p)^{N-1-j} \quad j=0, \ldots, N-1
$$

When a given mobile is at the infostation with $J=j$ neighbors, the probability $\beta^{\prime}$ that the given node is chosen for the infostation download is $1 /(j+1)$. Averaged over all $J$, the probability the given node is chosen for the download is

$$
\beta^{\prime}=\sum_{j=0}^{N-1} \frac{1}{j+1} P[J=j]=\frac{1-(1-p)^{N}}{N p}
$$

Similarly, when a node is at a regular location with $J=j \geq 1$ other neighbors present, 2 out of $j+1$ nodes are randomly chosen. The conditional access probability that a given node is one of the two chosen nodes is $2 /(j+1)$. Thus,

$$
\begin{aligned}
\beta & =\sum_{j=1}^{N-1} \frac{2}{j+1} P[J=j] \\
& =\frac{2\left[1-(1-p)^{N}-N p(1-p)^{N-1}\right]}{N p}
\end{aligned}
$$


Based on (4), the optimal $N$ is around $2 L$. Below, in equation (12), a more careful optimization of $\beta(N)$ in the limit of large $N, L$ with fixed density $\rho \triangleq N / L$, reveals that $\rho_{\text {opt }} \simeq 1.8$. One can use this result to determine the optimal spatial density of fixed infostations based on the anticipated spatial density of mobile subscribers.

When nodes $i$ and $j$ have the opportunity to exchange files, the probability of exchange $P_{E}$ depends on the files each node is holding. Suppose nodes $i$ and $j$ have $l_{i}$ and $l_{j}$ files in their caches. An exchange between the nodes will occur unless one node has a collection of files that is subset of the other's collection. Assuming, without loss of generality, that $l_{i} \leq l_{j}$, an exchange failure occurs if node $i$ chooses its subset of $l_{i}$ files out of the $l_{j}$ files of node $j$. Since there are $\left(\begin{array}{l}K \\ l_{i}\end{array}\right)$ total ways for node $i$ to choose its files, the probability of exchange is

$$
P_{E}\left(l_{i}, l_{j}\right)=1-\frac{\left(\begin{array}{c}
l_{j} \\
l_{i}
\end{array}\right)}{\left(\begin{array}{c}
K \\
l_{i}
\end{array}\right)} \quad 0 \leq l_{i} \leq l_{j} \leq K
$$

From (5), we can derive a tight upper bound for the probability $P_{E^{c}} \triangleq 1-P_{E}$ of no file exchange between neighbor nodes with $l_{i}$ and $l_{j}$ files such that $a K \leq l_{i} \leq l_{j} \leq(1-a) K$ and $0<a<1 / 2$. When $K$ is large such that $a K,(1-a) K$, and $(1-2 a) K$ are all much greater than 1 , an asymptotic upper bound $\tilde{P}_{E^{c}}$ for $P_{E^{c}}$ coincides with the Stirling's approximation for $P_{E^{c}}$ and is given by

$$
\ln \tilde{P}_{E^{c}}=[2(1-a) \ln (1-a)-(1-2 a) \ln (1-2 a)] K
$$

As the multiplier of $K$ is negative for $0<a<1 / 2$, we deduce that when $0<a<1 / 2$,

$$
\lim _{K \rightarrow \infty} P_{E}\left(l_{i}, l_{j}\right)=1, \quad a K \leq l_{i} \leq l_{j} \leq(1-a) K
$$

That is, if each node has a non-vanishing fraction of all $K$ files, a file exchange almost certainly will occur when the number of files in the system is large.

To find an upper bound for $P_{E^{c}}$ that is valid for most values of $l_{i}$ and $l_{j}$, we observe that the small $x$ approximation $\ln (1+$ $x) \simeq x$ implies

$$
\ln \tilde{P}_{E^{c}} \simeq-2 a^{2} K,
$$

implying that $P_{E^{c}}$ can be made arbitrarily close to zero by choosing $a>O(1 / \sqrt{K})$. When the number of files in the system is large, file exchange almost always happens among neighbors during most of the file dissemination process. In practice, we can regard $P_{E}=1$ when $K \geq 1000$. We will come back to this point when we discuss our simulation results in Figure 3.

In the following, we derive the expected networking time $E\left[T_{4}\right]$ for a node to obtain all files and the associated throughput $C_{4}$. We assume that $K$ is large such that (7) holds and we model the dynamics of movie downloading by the discrete time Markov chain illustrated in Figure 2. Denote the state as

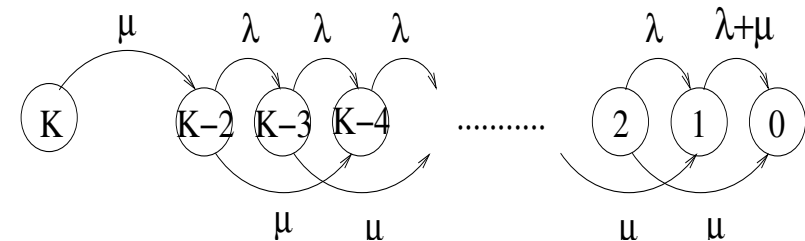

Fig. 2. Illustration of the Markov chain model. The shown values denote the state transition rates. Note that the depiction of self transitions is omitted.

the number of files remaining to be downloaded to a node. Initially a node is at state $K$. Since the first two files must be obtained from an infostation, the next state is $K-2$. Subsequently, in states $k \in\{1, \ldots, K-2\}$, each unit of time allows the following possibilities:

- With probability $p$, the node encounters the infostation and then with probability $\beta^{\prime}$ downloads two files. The state goes from $k$ to $k-2$ with probability $\mu=p \beta^{\prime}$.

- With probability $1-p$, the node is at a regular location and then with probability $\beta$ participates in a file exchange. The state goes from $k$ to $k-1$ with probability $\lambda=$ $(1-p) \beta$.

- With probability $1-\lambda-\mu$, no new files are obtained and the state stays the same.

Denote the expected first passage time from state $i$ to state 0 as $g_{i}$, where $(2 \leq i \leq K-2)$. Conditioning on the next state transition and rearranging yields the difference equation,

$$
g_{i}=\frac{1}{\lambda+\mu}+\frac{\lambda}{\lambda+\mu} g_{i-1}+\frac{\mu}{\lambda+\mu} g_{i-2}
$$

where the boundary conditions are given by $g_{0}=0$ and $g_{1}=$ $1 /(\lambda+\mu)$. Using $\mathrm{z}$-transforms, we solve (9) to obtain

$$
g_{i}=\frac{i(\lambda+2 \mu)+\left(1-\left(\frac{-\mu}{\lambda+\mu}\right)^{i}\right) \mu}{(\lambda+2 \mu)^{2}}
$$

It is obvious that $E\left[T_{4}\right]=1 / \mu+g_{K-2}$, where $1 / \mu$ is the expected time until a node first encounters the infostation and obtains the first two files.

For a network with a single infostation supporting $N$ nodes over $L$ locations, we consider the large-system and many-files regime in which $N, L, K \gg 1$ while the spatial density of nodes $\rho \triangleq N / L$ is held constant. In this regime, (2) and (4) imply that the infostation download probability and the conditional access probability converge to

$$
\begin{aligned}
\beta^{\prime}(\rho) & \sim \frac{1-e^{-\rho}}{\rho} \\
\beta(\rho) & \sim \frac{2}{\rho}\left(1-(\rho+1) e^{-\rho}\right)
\end{aligned}
$$

Furthermore, $\lambda \sim \beta(\rho)$ and $\mu \sim \beta^{\prime}(\rho) / L$ and the asymptote of the expected time for an arbitrary node to collect all $K$ files is

$$
E\left[T_{4}\right] \sim \frac{K}{\beta(\rho)}+\frac{L}{\beta^{\prime}(\rho)}
$$


Here, the second term is equal to $1 / \mu$ to account for the time for a node to fetch the first two files in an infostation encounter. The first term is an approximation to $g_{K-2}$ by assuming all remaining files are obtained from node to node file exchanges when infostation density is low, i.e. $L \gg 1$. If we further allow $K$ to grow large relative to both $N$ and $L$, the corresponding throughput $C_{4}$ of a node is

$$
C_{4}=\frac{K}{E\left[T_{4}\right]} \sim \beta(\rho), \quad \frac{K}{N}, \frac{K}{L} \rightarrow \infty
$$

We observe that the node density $\rho$ that maximizes $\beta$ also minimizes the expected networking time $E\left[T_{4}\right]$ and maximizes the throughput $C_{4}$.

To appreciate the extent to which social contract improves the rate of file dissemination of a completely noncooperative network, in which the only mechanism for file distribution is direct downloading from fixed infostations, we consider the Markov chain model for the latter. The corresponding difference equation for the first passage time from state $i$ to 0 is $g_{i}=1 / \mu+g_{i-2}$ for $i \leq K-2$, yielding $E\left[T_{4}\right]=g_{K}=$ $K L / 2 \beta^{\prime}$ and

$$
C_{4}=\frac{2 \beta^{\prime}(\rho)}{L}
$$

Hence, the social contract provides an $O(L)$, or equivalently $O(N)$ since $L$ and $N$ are of the same order, improvement to the individual file collection rate. The key ingredient in this improvement is the increase from $O(1)$ file deliveries per unit time made by an infostation to $O(N)$ peer-to-peer file exchanges per unit time. With more complex models for radio communication and user mobility, in particular those employed in [5], the ability to support $O(N)$ communication links in a population of $N$ mobile nodes should yield similar improvements.

The social contract also leads to a similar improvement to the dissemination rate considered in our simulations, defined as the rate at which files are collected by nodes through either downloading from fixed infostations or file exchanges. Since the individual file collection rate $C_{4}$ is $\beta$, file dissemination rate with social contract is $N \beta$ during most of the dissemination process. On the other hand, the file downloading rate at an infostation is 2 if a node is present there, thus file dissemination rate without social contract is slightly less than 2. Therefore, the improvement offered by the social contract is of the order $N$.

\section{Simulation Results}

In this section, we examine the impact of the number of nodes $N$ and number of files $K$ in the system on the network performance, evaluated in terms of the expected networking time $E\left[T_{i}\right]$ and throughput $C_{i}$. In our simulations, the network size is kept constant at $L=25$ nodes. A node moves to one of the neighbor locations w.p. $q=0.25$ at each unit time. The performance metrics are obtained from ensemble averaging over 100 simulations.

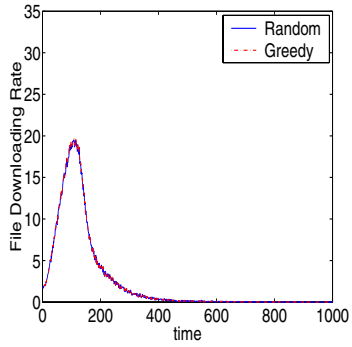

(a)

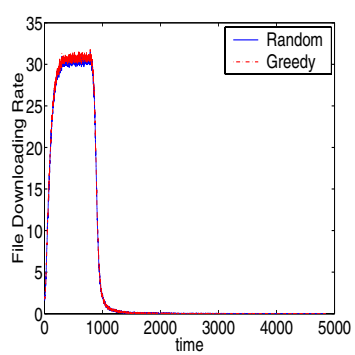

(c)

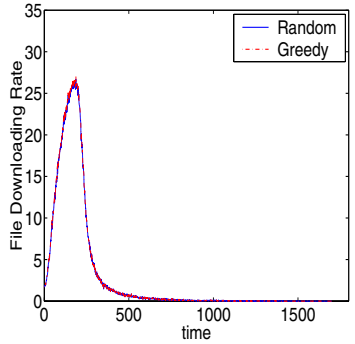

(b)

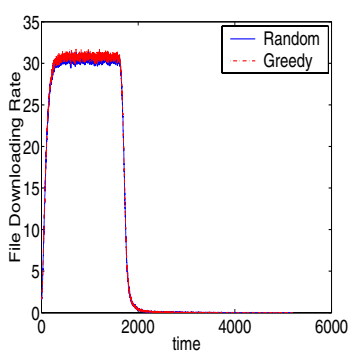

(d)
Fig. 3. Average number of files obtained at each unit time over 100 simulations. (a) $\mathrm{K}=50$, (b) $\mathrm{K}=100$, (c) $\mathrm{K}=200$, (d) $\mathrm{K}=500$.

For performance evaluation, we define the dissemination rate as the total number of files obtained, either by download from the infostation or by file exchange, per unit time over all mobile nodes. Figure 3 shows the dissemination rate averaged over 100 simulations runs. The number of nodes is held constant at $N=50$ and the number of files is varied $(K=50,200,500,1000)$. In all cases, the differences between the random and the greedy strategies were found to be very small. Thus, the random strategy is a good alternative to the greedy strategy for practical implementation.

From Figure 3, the y-intercept is slightly less than 2. Since the node density is high, it is probable to find at least a node at an infostation location and download 2 files at $t=0$. The file dissemination process has three distinct phases. In the first phase, the infostation seeds the mobile nodes with files and the dissemination rate increases rapidly as nodes obtain the ability to exchange files. Once most nodes have visited the infostation, $P_{E} \simeq 1$ and the dissemination rate remains steady at a peak rate that is a function of the access probability $\beta(\rho)$. In particular, each node will exchange one file with probability $P_{E} \beta(\rho) \simeq \beta(\rho)$. Over all $N$ nodes, the dissemination rate is $N \beta(\rho)$. Once a node has acquired all $K$ files, the social contract dictates that the node refrain from file exchanges. As the number of nodes with all $K$ files becomes significant, we enter the third phase in which the dissemination rate declines to zero as time evolves. The remaining nodes must download their files directly from an infostation, prolonging the time to download the entire movie. For all values of $K$, our simulations exhibit a significant tail associated with this final phase of dissemination.

As mentioned in the last section, in the absence of node 
to node file exchanges, the rate of file downloading shown in Figure 3 would have been constantly the y-intercept value of about 2, as opposed to $N \beta(\rho)$ most of the time. The simulation results are consistent with the analysis in the last section. As $P_{E} \simeq 1$ for large $K$, in each unit of time, each node will obtain one file with probability $\beta(\rho)$. With $N$ nodes in total, the average dissemination rate in the middle phase is $N \beta(\rho)$. In Figure 3, $N=50, L=25$, yields $\rho=N / L=2$ and the middle phase dissemination rate is very close to $N \beta(2) \simeq 30$ files per unit time. The ratio of this rate to that of the completely noncooperative network is about 15-a dramatic improvement. Incidentally, we can interpret Figure 3 as a scaled version of $P_{E}$ as a function of $t$. When $t \rightarrow 0$, most nodes have nothing in their caches, thus $P_{E}(t) \simeq 0$. Similarly, $P_{E}(t) \simeq 0$ when $t$ is large since most of the nodes have finished downloading everything.

Lastly, for a finite population of nodes, we can mark the boundaries of the middle phase by the times about which all nodes have $O(\sqrt{K})$ and $O(K-\sqrt{K})$ files, based on the discussion of the upper bound of $P_{E^{c}}$ after (8). We hence observe that the first and third phases require $O(L \sqrt{K})$ time, roughly on the order of the time required for each node to acquire $\sqrt{K}$ file solely by visiting the infostation. On the other hand, in the middle phase, the system must deliver $O(N K)$ files in total at a dissemination rate of $N \beta(\rho)$ files per unit time, and this requires $O(K)$ time. As $K$ increases (with $N, L$ fixed although not small), this middle phase comes to dominate the total dissemination time. Hence, for large $K$, the average dissemination rate is effectively the same as the peak dissemination rate of the middle phase. In short, as $K \rightarrow \infty$, the curve of Figure 3 converges to a rectangle with a constant file dissemination rate of $N \beta(\rho)$ files per unit time for a duration of $K / \beta(\rho)$ time units. This conclusion is consistent with the observation that the peak dissemination rate $N \beta(\rho)$ is simply $N$ times the average per node capacity $C_{4}$. We note that as $K \rightarrow \infty$, the transmission of each channel is only limited by contention, indicating the noncooperation strategy achieves almost optimum resource utilization.

In Figure 4, the networking time $T_{i}, i=1,2,3$ are plotted against the number of nodes $N$. The number of files is kept constant at $K=200$. From (2), it is easily verified that $\beta(\rho)$ is maximized at $\beta=1.7933$ users/location, or $N_{\mathrm{opt}}=45$ users over $L=25$ locations. This agrees with our observation in Figure 4(a), confirming that $N \simeq 45$ also minimizes $E\left[T_{1}\right]$. When $N$ increases past $N_{\text {opt }}, E\left[T_{1}\right]$ increases due to the increased contention at each location; however, the increase is partially offset by the increased opportunity for exchanges; hence, $E\left[T_{1}\right]$ is fairly insensitive to $N$ when $N \geq N_{\text {opt }}$. When $N<N_{\text {opt }}, E\left[T_{1}\right]$ increases quickly for decreasing $N$. When $N$ is small and node density is low, the system performance is hampered by the limited availability of file exchanges. In this case, $E\left[T_{1}\right]$ is very sensitive to $N$ since a small increase in $N$ significantly increases the rate of file exchange.

In Figure 4(b),(c), the optimum number of nodes that minimizes the networking time $T_{2}$ and $T_{3}$ are respectively

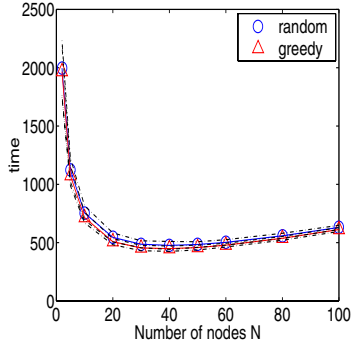

(a)

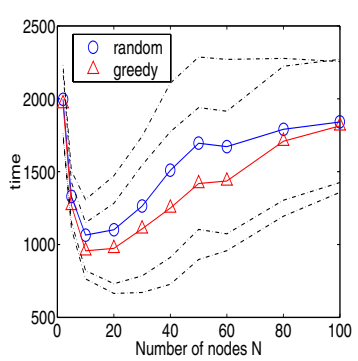

(c)

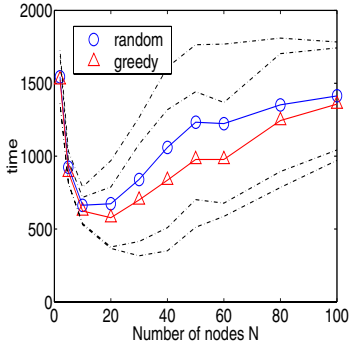

(b)
Fig. 4. Average networking time vs. the number of nodes $N$. (a) $E\left[T_{1}\right]$ when $80 \%$ of all nodes obtain all files, (b) $E\left[T_{2}\right]$ when all nodes obtain $80 \%$ of all files, (c) $E\left[T_{3}\right]$ when all nodes obtain all files.

$N_{\text {opt }}=20$ and $N_{\text {opt }}=10$ nodes, rather than $N=45$ nodes. This disparity arises from the observation in Figure 3(a),(b) that when $K$ is not large, the total download time depends strongly on the duration of phase three which has a long tail. The tail length depends largely on the rate at which mobile nodes can download from the infostation. The tail decreases as $N$ decreases because fewer nodes results in each node having better access to the infostation. On the other hand, $T_{1}$ is unaffected by the long tail. A plausible reason is that networking is unfair; $80 \%$ of the nodes finish downloading all files well before hitting the long tail regime.

With reference to Figure 5, the networking time is plotted against the number of files $K$ cached in an infostation. It is obvious that the networking time $T_{i}, i=1,2,3$ could be fitted to an asymptote as $K \rightarrow \infty$. The variance for $E\left[T_{1}\right]$ is small, indicating that the networking effect due to node mobility is deterministic. The slope of the asymptote is found to be around 1.63 , which is equal to $1 / \beta(N) . E\left[T_{2}\right]$ and $E\left[T_{3}\right]$, on the other hand, exhibit larger variances. The slope of the asymptotes for $E\left[T_{2}\right]$ and $E\left[T_{3}\right]$ are 1.1 and 1.6. When $K \leq 500$, we observe that $E\left[T_{2}\right]$ is larger than $E\left[T_{1}\right]$. Beyond $K=500$, $E\left[T_{2}\right]$ is smaller than $E\left[T_{1}\right]$. This demonstrates that as $K$ increases, the networking between the nodes is more fair. That is, all nodes have approximately the same file downloading time. A plausible reason is that $P_{E} \rightarrow 1$ as $K$ increases. The downloading rate is no longer influenced by individual file content, but depends primarily on mobility and contention. For large $K \geq 500$, the downloading time is long compared with the time scale of mobility ergodicity. Each node therefore has a downloading time that is almost the same, such that 


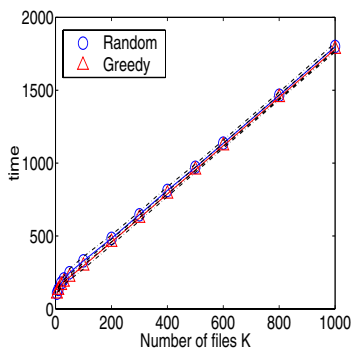

(a)

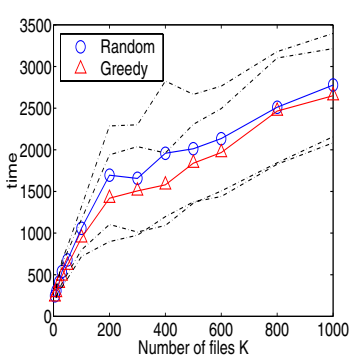

(c)

Fig. 5. Average networking time vs. the number of cached files $K$. (a) $E\left[T_{1}\right]$ when $80 \%$ of all nodes obtain all files, (b) $E\left[T_{2}\right]$ when all nodes obtain $80 \%$ of all files, (c) $E\left[T_{3}\right]$ when all nodes obtain all files. The dashed lines denote the 1 standard deviation upper and lower bounds from the mean value.

$E\left[T_{1}\right]>E\left[T_{2}\right]$.

\section{DAta DiVersity}

In Figure 5, we showed that the networking time $E\left[T_{i}\right], i=$ $1,2,3$ can be fitted nicely to an asymptote as $K$ increases. The corresponding throughputs are plotted in Figure 6 versus $K$. We observe that the throughput is an increasing function of $K$. It is instructive to find the asymptotic value of throughput $C_{i}^{\infty}$ as $K \rightarrow \infty$. To do this, we use the intuition captured in (13) and approximate the asymptote of $T_{i}$ by

$$
T_{i}^{\infty}=m_{i} K+c_{i}
$$

where $m_{i}$ is the slope and $c_{i}$ is the vertical intercept. Since the asymptote $T_{i}^{\infty}$ approaches $E\left[T_{i}\right]$ arbitrarily close when $K \rightarrow \infty$, we compute the asymptotic capacity as

$$
C_{i}^{\infty}=\lim _{k \rightarrow \infty} \frac{K}{T_{i}}=\lim _{k \rightarrow \infty} \frac{K}{T_{i}^{\infty}}=\frac{1}{m_{i}}
$$

Recall that $m_{3}=1.63$ as read from Figure 5(c). Thus $C_{3}=$ 0.613 files per node per unit time, or 30.65 files per unit time in our network where $N=50$. This agrees with our result in Figure 3(d). When $P_{E} \simeq 1$, the rate for data dissemination is around 30 files per unit time. Incidentally, we observe that

$$
\lim _{K \rightarrow \infty} C_{3}=\lim _{K \rightarrow \infty} C_{4}
$$

When $K \rightarrow \infty$, networking is fair and each node has the same throughput asymptotically. Thus, our simulation results are consistent with our simplified analysis.

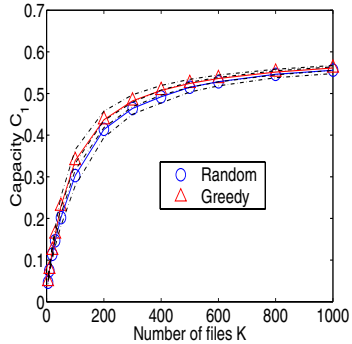

(a)

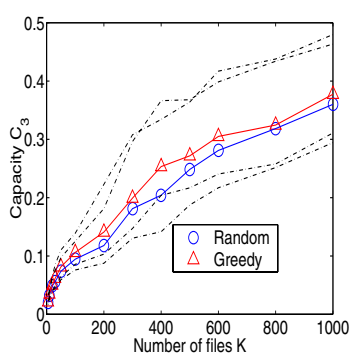

(c)

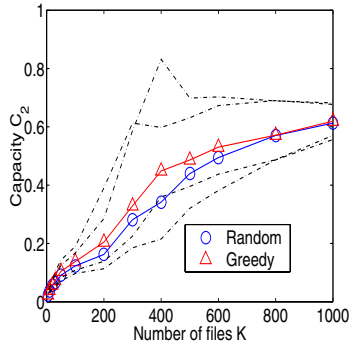

(b)
Fig. 6. Throughput capacity vs. the number of cached files $K$. (a) $C_{1}$ when $80 \%$ of all nodes obtain all files, (b) $C_{2}$ when all nodes obtain $80 \%$ of all files, (c) $C_{3}$ when all nodes obtain all files. The dashed lines denote the 1 standard deviation upper and lower bounds from the mean value.

The apparent increase in throughput can be understood using the concept of data diversity. In wireless communications diversity refers to the exploitation of variations in signal strength due to multipath fading. Since multipath fading exhibits signal variations over spatial, time and frequency domains, diversity techniques can be applied to select the strongest signal component over the respective domains. Diversity can also be exploited in a more general sense. In multiuser diversity, for instance, a receiver exploits the variability of received signal strength over different mobile nodes, and selects the node with the best channel for transmission.

Whereas the above techniques belong to the category of communication diversity, we argue that a new form of diversity, coined data diversity, is exhibited in noncooperative content distribution. When nodes are not cooperating, each node effectively has a preference list of files that evolves with time. If the number of disseminated files is large, there are more selections from a node's perspective. (5) and (7) dictate that file dissemination under the social contract is more efficient when there are more selections available for each node. There are, however, some differences between receiver diversity and data diversity. We note that receiver diversity is the result of a passive environment and we can exert no influence to the outcome. Data diversity, on the other hand, is the consequence of our social contract, over which we have complete control. Nevertheless, the social contract provides a general framework to study non-cooperation content distribution in mobile infostation networks. We have shown that data diversity is relevant to noncooperative data dissemination, which is gaining more attention in the networking community. 
Data diversity may also have implications to other peer to peer networks other than mobile infostation networks such as content distribution on the wired Internet.

Consider the possibility that several content providers use the mobile infostation infrastructure to disseminate their content (that are not highly overlapping) to a common group of subscribers. If a subscriber has files from content provider $\mathrm{A}$ and he encounters another subscriber with files from content provider B, these files generally would not be interexchangeable since they originated from different content providers. However, our results point out that content distribution for each provider would be more efficient, in terms of both throughput and fairness, if there were mutual agreements between content providers such that all files are interexchangeable, effectively increasing the content size $K$.

On the other hand, even if the content providers do not collude in data dissemination, data diversity can still be useful, say, in the dissemination of a single movie of a movie distribution network. Consider the scenario when a DVD quality movie is disseminated in a highway infostation network populated with fast vehicular subscribers. A typical drivethrough infostation has a coverage radius of $20 \mathrm{~m}$ [4]. A vehicle at a speed $20 \mathrm{~m} / \mathrm{s}$ therefore has a connection time of 2 seconds when it is in the coverage area of an infostation. Similarly, for two vehicles moving in opposite direction, the connection time is only 1 second. Suppose the infostation radios operate at a modest data rate of $160 \mathrm{Mbit} / \mathrm{s}$ (which still substantially outperform the state of the art $54 \mathrm{Mbit} / \mathrm{s} 802.11 \mathrm{a}$ access points available today). In order to facilitate the file exchange of two data files in the worst case of a head-on mobile to mobile encounter, the file size should be no more than 10MByte. On the other hand, the typical size of a DVD quality movie is roughly 5 GByte. Thus, a movie should be split into $K=500$ files and cached in fixed infostations for dissemination. Our simulation results in Figure 6(c) have shown that with a modest content size of $K=500$ files, the achievable per node capacity $C_{3}$ is $80 \%$ of the theoretical per node capacity $\lim _{K \rightarrow \infty} C_{4}$ for asymptotically large $K$. Thus, without even relying on the collusion between the content providers, we can enjoy the benefits of data diversity in the dissemination of a single movie.

\section{DISSIMILAR INTERESTS}

In our basic model, we assume all nodes have a common interest in $K$ files. In this section, we extend the common interest model to the case where each node has interest in only a subset of the $K$ files cached in the infostation. Depending on the type of content, the interests of the nodes can be mutually exclusive or partially overlapping. For instance, suppose multiple movies, say $1 / \alpha$ movies are cached in the infostations, where $0<\alpha \leq 1$. Each movie has the same length and is divided into $\alpha K$ files. If each node is interested in one movie only, then any two nodes will have interests that are either exactly the same or mutually exclusive. More generally, the interests of all nodes are partially overlapping.
Consider the case where multiple TV shows are cached in the infostations. Without loss of generality we assume each TV show is stored as one file. Each node is interested in $\alpha K \mathrm{TV}$ shows or files that is randomly selected from all $K$ cached files.

Recall in section II that a user strategy consists of two parts. Suppose two nodes seize the local channel successfully. First the two nodes must determine whether to exchange files. Second, upon an agreement of performing a file exchange, each node determines what to exchange as specified by the random or greedy strategy. In the common interest model, each node is interested in every file cached in the infostations. A node therefore is genuinely interested in every file that it does not have. In the dissimilar interest model, however, the above assumption is no longer valid. We can differentiate two user strategies in which neighbor nodes determine whether to exchange files. In user strategy $\mathbf{I}$, neighbor nodes $A$ and $B$ perform a file exchange only if both nodes discover a file of genuine interest on inspection of each other's caches. In user strategy II, nodes $A$ and $B$ are obliged to exchange files if each node has a file that the other node does not have, whether or not those files are of genuine interest.

Once the nodes agree on a file exchange, either the random or greedy downloading strategy can be used in both user strategies. Nevertheless, we have demonstrated through analysis and simulations in earlier sections that the random and greedy downloading algorithms have almost identical performance. Hereafter, we consider only the random downloading strategy when we compare the performance of user strategy I and II in the simulation studies.

We have performed simulations to study the network performance for the multiple movies model, where node interests are either exactly the same or mutually exclusive. The results for the TV show model are reported in the journal version of this paper. The network performance is evaluated in terms of $\alpha$, which characterizes the extent of overlapping interest with other nodes. When $\alpha$ is very small, each node is interested in a small fraction of all files. The interests of any two nodes are likely to be mutually exclusive. As $\alpha$ increases, more nodes are interested in the same files. It is therefore more probable for a node to run into another node that has the same interest. When $\alpha=1$, all nodes are interested in all $K$ files and our model reduces to the common interest model.

In our simulations, we assume the number of nodes in each infostation block is $N=40$ and the total number of files is $K=1000$. We consider the multiple movies model in which $1 / \alpha=1,2,4,5,10,20,40$ movies are distributed at the infostations. Each movie is split into $\alpha K$ files, and the corresponding values of $\alpha$ are $1,0.5,0.25,0.2,0.1,0.05,0.025$. In the case of 40 movies, each node is interested in different movies and have mutually exclusive interest. The number of nodes having the same interest increases with $\alpha$. When $\alpha=1$, all nodes have a common interest for the same movie. Denote $E\left[T_{i}^{\alpha, j}\right], i=1,2,3$ as the expected networking time of user 


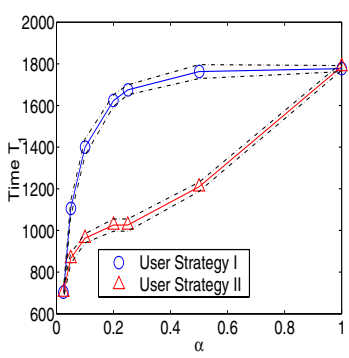

(a)

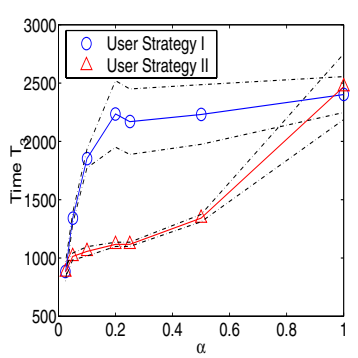

(c)

Fig. 7. Average networking time vs. the fraction of interested files $\alpha$. (a) $E\left[T_{1}\right]$ when $80 \%$ of all nodes obtain all files, (b) $E\left[T_{2}\right]$ when all nodes obtain $80 \%$ of all files, (c) $E\left[T_{3}\right]$ when all nodes obtain all files. The dashed lines denote the 1 standard deviation upper and lower bounds from the mean value.

strategy $j$, where $j=1,2$. We are interested in finding the expected networking time for both user strategies.

Referring to Figure 7, the networking time of both user strategies is plotted versus $\alpha$. We observe that even when $\alpha$ is very small, the downloading time of user strategy I is quite large. In particular, when $\alpha=0.025$, the number of files wanted by each node is only $\alpha K=25$. The corresponding expected networking time $E\left[T_{i}^{\alpha}\right], i=1,2,3$ for both user strategies is approximately 700,750 , and 850 units. At $\alpha=0.025$, each file is desired by one node. This is easily seen since by symmetry, each file is desired by $\alpha N=(0.025)(40)=1$ node. Suppose all nodes observe user strategy I. It is obvious there is no file exchange between nodes since each node keeps only files that is wanted by that particular node only. On the other hand, when user strategy II is used, file exchanges between nodes are allowed. Nevertheless, a node never fetches a file and benefits from a file exchange since all nodes have mutually exclusive interest. For both user strategies, each node has to download every desired file directly from an infostation. The absence of concurrent file exchanges in conjunction to infostation downloading explains the long and identical networking time.

Referring to Figure 7 again, it is obvious that $E\left[T_{i}^{\alpha, 1}\right]$ and $E\left[T_{i}^{\alpha, 2}\right]$ are increasing with $\alpha$ for $i=1,3$. This is plausible since in general, more time is needed for a fraction of nodes to finish file downloading as the number of desired files increases. An interesting (although not statistically significant) exception is observed for $E\left[T_{2}^{\alpha, 1}\right]$, and might be explained by the following. When the number of files $\alpha K$ to be downloaded is small, a node usually runs into other nodes that have mutually exclusive interests. The node therefore has to download most of the files directly from the infostations, unable to enjoy the benefit of spatially concurrent file exchanges. As a result, these nodes have a large networking time. As $\alpha$ increases further, most, if not all, of the nodes participate in beneficial file exchanges due to the presence of nodes with the same interests. Since $E\left[T_{2}^{\alpha, 1}\right]$ is dominated by the nodes without file exchanges when $\alpha$ is small, this explains the peak at $\alpha=0.2$.

In order to explain the increasing trend of networking time with $\alpha$, and to characterize the performance difference for both user strategies, we examine the mechanism of the data dissemination in the following. As $\alpha$ increases from $\alpha=$ 0.025 , there is more nodes with the same interests. Each file is desired by $\alpha N$ users on average. Consider user strategy I. Approximately $\alpha N$ nodes are willing to act as the networking agents for each file and possibly carry the file in their cache as these nodes roams around the network. When $\alpha$ gets larger, the number of networking agents for each file increases. Since the circulation of a particular file is constrained by the number of networking agents for that file, increasing $\alpha$ effectively promotes the circulation of each file. This impacts the number of node-to-node file exchanges favorably, allowing more simultaneous file exchanges to take place. Consequently, the networking time $E\left[T_{1}^{\alpha, 1}\right]$ and $E\left[T_{3}^{\alpha, 1}\right]$ flatten quickly as $\alpha$ is increased.

For user strategy II, the networking time is consistently smaller than that of user strategy I as $\alpha$ increases from 0.025 . Although nodes have little overlap of common interests when $\alpha$ is small, user strategy II dictates that a file exchange ensues whenever each node can retrieve a file that it does not have on inspection of the cache of the other node. Thus, all $N$ nodes are willing to act as the networking agents for all files. The circulation of each file is not constrained by the particular interests of each node. Since nodes are more admissable and willing to carry files in user strategy II, the networking time is consistently smaller.

In the case $\alpha=1$, our dissimilar interest model reduces back to the common interest model. Both user strategies I and II have identical networking time $E\left[T_{i}^{\alpha}\right], i=1,2,3$, that agrees to the corresponding values $E\left[T_{i}\right], i=1,2,3$ for the common interest network model. When $K$ is reasonably large (in our case $K=1000$ ), data diversity dictates that $P_{E} \rightarrow$ 1 and the networking time is then only constrained by the contention probability $\beta$ given by (13).

\section{Multiuser Diversity}

In Figure 7, we showed that the networking time $E\left[T_{i}^{\alpha}\right], i=$ $1,2,3$ for user strategy II is always less than that of user strategy I. The corresponding network capacity is plotted versus $\alpha$ in Figure 8. Again, x-axis denotes the fraction $\alpha$ of files that each node is interested in, where $\alpha$ takes the values of $0.025,0.05,0.1,0.2,0.25,0.5,1$. We observe that for both user strategies, the network capacity $C_{i}^{\alpha}, i=1,2,3$ is 


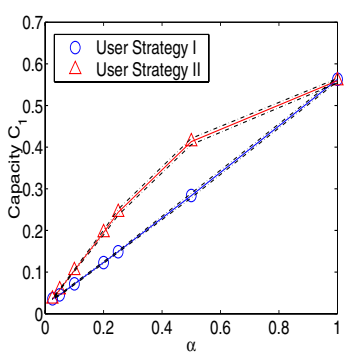

(a)

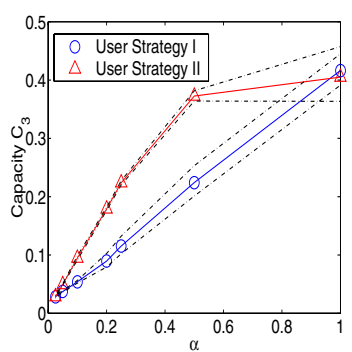

(c)

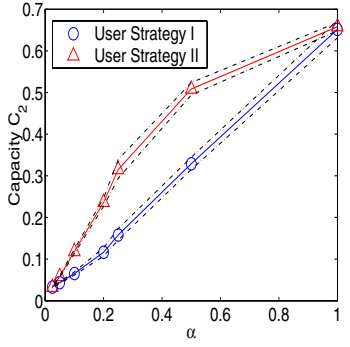

(b)
Fig. 8. Throughput capacity vs. the fraction of interested files $\alpha$. (a) $C_{1}$ when $80 \%$ of all nodes obtain all files, (b) $C_{2}$ when all nodes obtain $80 \%$ of all files, (c) $C_{3}$ when all nodes obtain all files. The dashed lines denote the 1 standard deviation upper and lower bounds from the mean value.

strictly increasing with $\alpha$. The capacity of user strategy II is consistently larger than that of user strategy I when nodes have dissimilar interests $\left(\frac{1}{N}<\alpha<1\right)$. The capacity of both strategies coincide when $\alpha \leq \frac{1}{N}$ and $\alpha=1$. When $\alpha \leq \frac{1}{N}$, all nodes have mutually exclusive interests. Even though user strategy II allows node-to-node file exchanges, there is no corresponding gain in network capacity. Similarly, when $\alpha=1$, our model reduces back to the common interest model. Thus both user strategies I and II have almost identical capacities.

The increasing trend of network capacity with $\alpha$ can be understood using the concept of multiuser diversity inherent to mobile infostation networks. The efficiency of dissemination of this file is dependent on the willingness of the mobile nodes to carry it across the network. If a node is willing to carry a particular file, then the node is effectively acting as a networking agent for that file. For user strategy I, each file is wanted by approximately $\alpha N$ nodes, who are willing to act as the networking agents for the file. For strategy II, each node is obliged to carry every file even if the file is not wanted by the node. The number of networking agents is then equal to the number of nodes $N$ irrespective of $\alpha$. We argue that the performance improvement of user strategy II is an exploitation of multiuser diversity, where the number of nodes willing to act as networking agents for each file is increased. Since the circulation of a particular file is equal or less than the number of networking agents for that file, the actual circulation of each file improves as the number of networking agents increases. As a consequence of improved file circulation, the efficiency of file exchanges improves as stipulated by data diversity, allowing multiple spatially concurrent file exchanges to take place.

From the above argument, we expect the two user strategies have the greatest performance disparity when $\alpha$ is small. Figure 8, however, shows that the percentage performance disparity is maximum when $\alpha$ is about 0.5 . We note that the increase of the number of networking agents indeed leads to a proportional increase in the number of files in circulation. However, when $\alpha$ is small, each file is of genuine interest to only a few nodes and most file exchanges involve files that are of no interest to either node. Thus even if the circulation of all files is increased significantly, the corresponding increase in the number of file exchanges is not beneficial.

There are two opposing factors that impact the performance of user strategy II. For small $\alpha$, the number of networking agents for user strategy II is increased dramatically by a factor of $1 / \alpha$. However, most of the file exchanges are not beneficial since node interests are largely non-overlapping. For large $\alpha$, there is only a nominal increase in the number of networking agents. However, since most nodes have very similar interests, each node gets many desired files and benefits from file exchanges. Our simulation results show that for $\alpha=0.5$, we achieve an attractive, and perhaps optimum, tradeoff in terms of capacity gain. The corresponding capacity $C_{i}^{\alpha, 2}, i=1,2,3$ improvement of user strategy II over user strategy I is above $66 \%$ for all three cases.

Consider a movie distribution network in which 20 movies are cached in the infostations, making a total of $K=1000$ cached files. Suppose each node is interested in only one movie of 50 files. This is equivalent to our multiple movies model with $\alpha=0.05$. If all nodes observe user strategy I, the networking time $E\left[T_{i}^{\alpha, 1}\right]$ is respectively 1100,1200 and 1300 units. On the other hand, if all nodes observe user strategy II, the networking time $E\left[T_{i}^{\alpha, 2}\right]$ is 825,825 and 1000 units, roughly $70 \%$ of the original time. In content distribution, usually each node wants to minimize the networking time for files of genuine interest. Our simulation results point out that if a node acts as a networking agent for files he is not interested in, it actually expedites the file downloading process, reducing the networking time while enjoying a network capacity gain as warranted by multiuser diversity. This is an interesting result because it implies each node has an incentive to act as a networking agent and assist in data dissemination without having an explicit node cooperation model.

Although the exploitation of multiuser diversity in user strategy II yields better network capacity, it comes at a cost of increased energy consumption due to more frequent file exchanges. Thus there is a tradeoff between energy consumption and network capacity. If the network nodes have plentiful energy reserves, say infostations on vehicles, they should adopt user strategy II to tradeoff energy consumption for better throughput capacity. On the other hand, for nodes having scanty energy supply, they can cut down the energy consumption by sacrificing some throughput. Moreover, nodes do not need to adopt the same user strategy in a network. Each 
node can independently decide what user strategy to adopt based on its current level of residual energy.

We note that in user strategy II, there is implicit cooperation between nodes. Each node is obliged to act as the networking agent for files that it is not interested in, That is, each node caches and disseminates personally uninteresting files for other nodes as it roams the network. The performance gain of user strategy II over strategy I agrees with the intuition that more cooperation usually leads to better system performance. Although user strategy II requires implicit cooperation between nodes, there is no corresponding control overhead due to user cooperation. We do not assume the exchange of files of genuine interest to neighbor nodes takes priority over other types of file exchanges. In our implementation, when there are multiple neighbor nodes at the same location, the first two nodes that broadcast control messages to request a file exchange seize the channel. This rule is equivalent to randomly picking two nodes from all neighbor nodes with no signaling overhead and is completely determined by contention. Note that giving priority to exchanges of files of genuine interest may improve overall system performance if one can develop an efficient protocol between multiple neighbor nodes to determine the optimal node pair to exchange files.

\section{CONCLUSION AND FURTHER WORK}

We have addressed the issue of noncooperation among nodes in the context of content distribution in mobile infostation networks. In the first part, we assume all nodes have a common interest of $K$ files cached in the infostations. We have shown that it is possible to drastically increase the rate of file dissemination of a completely noncooperative network by requiring the absolute minimal cooperation among users in the form of a social contract. A random and a greedy file downloading algorithms are examined and shown to have similar performance. We show that there exists some optimal node density in these networks such that the access probability of a node is maximized and the networking time is minimized. More importantly, we show that the total number of files cached in the infostations impacts the networking fairness and throughput. We identify this phenomenon as data diversity that is distinct from conventional communication diversity. When nodes are noncooperative and have individual preference on data, the network exhibits data diversity and the throughput of each node increases with increasing content variety. In the second part, we extend the common interest model to the case where nodes have partially overlapping but dissimilar interests. Two user strategies are considered for this model. We show in our simulations that a file exchange strategy that takes advantage of the multiuser diversity inherent in mobile infostations results in enhanced network performance. We conclude that both data diversity and multiuser diversity can be exploited in the mobile infostation architecture even if nodes are noncooperative.

In the present work, simple mobility and interference models are used to facilitate analysis. This approach has been fruit- ful, leading to the observations of two diversity phenomena in noncooperative content distribution. Nevertheless, a thorough examination of the implications of mobility and interference to the network performance of mobile infostations is called for. As a first step, the issue of interference modeling is addressed in a recent paper [14]. The effect of transmit range on network capacity is examined. We found out a stipulated transmit range improves the capacity of a mobile infostation network further. An optimal number of neighbors exists for mobile infostation networks that is distinct from the well known 6-8 magic number [9], [13], [8] for multihop ad hoc networks. Moreover, the network capacity is linearly increasing with node density. Thus mobile infostation is an attractive alternative to multihop networking in future pervasive computing environments, where high node density dooms the throughput of multihop networks. On the other hand, the effect of mobility on mobile infostations is currently being studied. The connection time in each node to node encounter obviously depends on node mobility and needs to be quantified. To this end we have proposed a sophisticated mobility model for highway mobile infostation networks that allows for performance analyses based on renewal and queuing theories. We conjecture that the performance of mobile infostation networks are robust to mobility.

\section{REFERENCES}

[1] J. Broch, D.A. Maltz, D.B. Johnson, Y.C. Hu, and J. Jetcheva. A performance comparison of multi-hop wireless ad hoc network routing protocols. In Proc. Mobicom, pages 85-97, 1998.

[2] S.R. Das, C.E. Perkins, E.M. Royer, and M.K. Marina. Performance comparison of two on-demand routing protocols for ad hoc networks. IEEE Personal Communications, 8(1):16-28, Feb. 2001.

[3] R. H. Frenkiel, B. R. Badrinath, J. Borras, and R. Yates. The infostations challenge: Balancing cost and ubiquity in delivering wireless data. IEEE Personal Communications, 7(2):66-71, April 2000.

[4] R. H. Frenkiel, B. R. Badrinath, J. Borras, and R. Yates. The infostations challenge: Balancing cost and ubiquity in delivering wireless data. IEEE Personal Communications, 7(2):66-71, April 2000.

[5] M. Grossglauser and D. Tse. Mobility increases the capacity of ad-hoc wireless networks. In Proceedings of IEEE INFOCOM '01, volume 3, pages 1360-1369, 2001.

[6] P. Gupta and P.R. Kumar. Critical power for asymptotic connectivity in wireless networks. Stochastic Analysis, Control, Optimization and Applications: A Volume in Honor of W.H. Fleming, pages 547-566, 1998.

[7] G. Holland, N. Vaidya, and P. Bahl. A rate-adaptive mac protocol for multi-hop wireless networks. In The seventh annual international conference on Mobile computing and networking, July 2001.

[8] T.C. Hou and V.O.K. Li. Transmission range control in multihop packet radio networks. IEEE Trans. Commun., 34(1):38-44, 1986.

[9] L. Kleinrock and J. Silvester. Optimum transmission radii for packet radio networks or why six is a magic number. In Proceedings of the IEEE National Telecommunications Conference, pages 4.3.1-4.3.5, December 1978.

[10] M. Papadopouli and H. Schulzrinne. Effects of power conservation, wireless coverage and cooperation on data dissemination among mobile sevices. In Proc. IEEE MobiHoc 'O1, 2001.

[11] R. Ramanathan and Rosales-Hail. Topology control of multihop wireless networks using transmit power adjustment. In Proc. IEEE INFOCOM '00, pages 404-413, 2000.

[12] S.M. Ross. Stochastic Processes. John Wiley \& Sons, New York, 1983.

[13] H. Takagi and L. Kleinrock. Optimal transmission ranges for randomly distributed packet radio terminals. IEEE Trans. Commun., 32(3):246257, March 1984.

[14] W.H. Yuen and R.D. Yates. Optimum transmit range and capacity of mobile infostation networks. submitted for publication. 